\title{
LA INTEGRACIÓN URBANA COMO PARTE DEL CONTENIDO DEL DERECHO A LA VIVIENDA ESTÁNDARES INTERNACIONALES Y RECONOCIMIENTO EN EL DERECHO CHILENO
}

\section{URBAN INTEGRATION AS PART OF THE CONTENT OF THE HOUSING RIGHT INTERNATIONAL STANDARDS AND RECOGNITION IN CHILEAN LAW}

\section{Alfonso Andres Henriquez Ramirez ${ }^{1}$ Víctor Moreno Toledo ${ }^{2}$}

Resumen: En el derecho internacional se establecen una serie de obligaciones, las cuales, en general, disponen que los Estados deben asegurar el buen uso del espacio urbano sobre una base de igualdad y no discriminación. Por otro lado, en el derecho chileno existen suficientes elementos para concluir que la integración urbana constituye un principio que obliga a los poderes públicos a adoptar medidas con el objeto de avanzar hacia una distribución del espacio urbano sobre una base más equitativa. El trabajo analiza este marco normativo, en función de los principios y directrices que se disponen para estos efectos en el derecho internacional.

Palabras clave: derecho a la vivienda, integración urbana, segregación residencial, derecho urbano.

Abstract: The instruments of international law establish a series of obligations which generally state that States must ensure the proper use of urban space on a basis of equality and non-discrimination. On the other hand, in Chilean law there are sufficient elements to conclude that urban integration constitutes a principle that forces public authorities to adopt measures in order to move towards a distribution of urban space on a more equitable basis. The paper analyzes this regulatory framework, based on the principles and guidelines that are available for these purposes in international law.

Keywords: right to housing, urban integration, residential segregation, urban law.

\footnotetext{
${ }^{1}$ Profesor Departamento Historia y Filosofía del Derecho; Universidad de Concepción; Profesor Asistente; Doctor en Derecho; Universidad de Concepción - Chile. Email: alfohenriquez@udec.cl

2 Profesor Departamento Departamento de Derecho Económico; Universidad de Concepción; Profesor Instructor, Magister en Derecho. Universidad de Concepción - Chile. E-mail: victormoreno@udec.cl
} 


\section{Introducción}

El derecho a la vivienda, se enmarca dentro de lo que hoy conocemos como derechos de tercera generación. Su contenido ha desbordado el solo acceso a la residencia habitacional, expandiéndose a la calidad de la misma, cuestión que abarca múltiples aspectos, entre ellos, su ubicación.

En la actualidad, nuestro problema no radica solo en la disminución de la pobreza urbana, materia en la cual han existido avances sustantivos según indicadores de aceptación generalizada. Hoy nos encontramos ante una dificultad mayor, la desigualdad, la cual se expresa en lo referente a nuestra investigación, en la cada vez mas escaza convivencia e interacción que se produce entre los distintos estratos sociales. Convivencia indispensable en la noción misma de lo que entendemos por Estado.

Esta falta de contacto social, tiene su explicación en la cada vez más patente segregación urbana. La cual, se ha visto favorecida por la aquiescencia estatal en la materia, manifestada en una vigorosa concepción de la propiedad individual, la institución del mercado como asignador eficiente de "todo" bien escaso, un modelo urbano de crecimiento por extensión de las ciudades, falta de alicientes a la densificación, falta de incentivos a la creación de espacio público, falta de políticas publicas integradoras, entre otras. Las cuales, en definitiva, perfilan las bases de un modelo urbano segregador.

Lo anterior, representa un gran desafío para nuestra sociedad, más aun cuando la vivienda aparece consagrada como un derecho "fundamental" y con una particular configuración, que importa un derecho para los ciudadanos y lo más importante, una obligación de tutela y goce por parte de los Estados.

\section{1.- La integración urbana como parte del contenido del derecho a la vivienda adecuada en el derecho} internacional de los derechos humanos (DIDH)

\section{1.- Consideraciones generales}

En el DIDH el derecho a la vivienda adecuada aparece reconocido de forma clara en el art. 11.1 del Pacto Internacional de Derechos Económicos Sociales y Culturales (PIDESC) en los siguientes términos: 
Los Estados Partes en el presente Pacto reconocen el derecho de toda persona a un nivel de vida adecuado para sí y su familia, incluso alimentación, vestido y vivienda adecuados, y a una mejora continua de las condiciones de existencia. Los Estados Partes tomarán medidas apropiadas para asegurar la efectividad de este derecho, reconociendo a este efecto la importancia esencial de la cooperación internacional fundada en el libre consentimiento.

Para entender correctamente el sentido de esta norma, y en especial, el alcance de las obligaciones que los Estados han asumido en relación al problema de la integración urbana, nos vamos a centrar a continuación en el análisis de los estándares que sobre el particular ha desarrollado el Comité sobre Derechos Económicos Sociales y Culturales (CDESC) en su Observación General N 4.

En este sentido, un aspecto que llama la atención, reside en que para el CDESC, la obligación básica que asume la administración, no consiste simplemente en asegurar el derecho a una vivienda, sino que en garantizar el derecho a una vivienda que satisfaga un cierto estándar de adecuación. A este respecto, el CDESC parte de la base que los Estados cumplen con este mandato, cuando a lo menos, garantizan los siguientes aspectos: seguridad de la tenencia, disponibilidad de servicios, materiales, facilidades e infraestructura, gastos soportables, habitabilidad, asequibilidad, lugar y adecuación cultural (CESCR, OBSERVACIÓN GENERAL N 4; 1991; ART. 11).

Tal como se aprecia, resulta bastante claro que el CDESC entiende el derecho a la vivienda de forma muy amplia, en la medida en que no lo circunscribe simplemente a la posibilidad de acceder a una morada, sino que también, supone: "el derecho de todo hombre, mujer, joven y niño a tener un hogar y una comunidad seguros en que puedan vivir en paz y dignidad" (ONU-KOTHARI M., 2008, PÁRR. 4; HOHMANN J., 2013, p. 20). En efecto, y por vía de ejemplo, el derecho en estudio abarca cuestiones típicamente relacionadas con el ejercicio de derechos de libertad, tales como la protección contra el desalojo forzoso, la destrucción arbitrarias del hogar o el derecho a elegir la residencia y el lugar donde vivir, otras, vinculadas con el respeto al principio de no discriminación, como es el caso del derecho a poder acceder a la vivienda en igualdad de condiciones en relación al resto de la población, otras, relacionadas con derechos de participación, como el derecho a intervenir en la adopción de decisiones vinculadas con el goce de esta garantía, y otras claramente vinculadas con el reconocimiento de derechos de prestación, como son las relativas al deber de asegurar que la vivienda proporcione protección contra el frio, la humedad, el calor, la lluvia, el viento u otros riesgos para la salud, que esta 
cuente con agua potable, energía para la cocción, calefacción y alumbrado, y, que se encuentre en un lugar que le permita a sus ocupantes poder acceder a oportunidades de empleo, servicios de salud, educación, guardería y otros servicios sociales (ONU-HABITAT, 2010, p. 3-4; PISARELLO G., 2013, p. 142 y ss.).

Para justificar esta doctrina, el CDESC trae a colación algunas reglas generales de interpretación que existen en materia de derechos humanos. Así, y en primer término, el Comité razona en base a la idea que los tratados sobre derechos humanos recogen un principio particular de interpretación: el principio pro persona, de acuerdo con el cual, se deben interpretar los enunciados que reconocen derechos, de la forma más amplia o extensiva posible, e inversamente, que se debe optar por el sentido más restringido, cuando sea necesario establecer límites al ejercicio de alguna garantía fundamental (PINTO M., 1997, p. 163; PÌDCP, 1966, ART. 5.2). En este contexto, no podemos olvidar que los tratados sobre derechos humanos tienen como objeto y fin la consagración de un conjunto de derechos y libertades indisponibles para sus titulares, de manera que cada vez que sea necesario determinar el contenido y alcance de un derecho especifico, el intérprete le deberá dar al enunciado a interpretar la mayor amplitud posible, a fin de asegurar precisamente que el mandato normativo establecido por la respectiva convención, alcance eficacia y vigencia práctica (NASH C.; NOGUIERA, 2013, p. 170; CONV. DE VIENA SOBRE EL DERECHO DE LOS TRATADOS, 1969, ART. 31). En segundo término, el CDESC también tiene a la vista el principio de indivisibilidad e interdependencia de los derechos humanos (CESCR, OBSERVACIÓN GENERAL N 4; 1991; ART. 11). De acuerdo con este principio, las garantías fundamentales constituyen un conjunto en el cual cada una de sus partes resulta necesaria para el adecuado goce y disfrute de las demás. Esto significa que los derechos humanos no se pueden entender de forma fragmentaria o como si fuesen compartimentos estancos, sino que de manera complementaria, es decir como elementos que se refuerzan recíprocamente (FERNÁNDEZ RUIZ-GÁLVEZ, 1996, p. 696.). A este respecto, el artículo 13 de la Declaración de Teherán de 1968, resulta contundente:

Los derechos humanos y las libertades fundamentales son indivisibles, resultando imposible el disfrute completo de los derechos civiles y políticos sin el de los derechos económicos, sociales y culturales. Los progresos duraderos en la vía de la aplicación de los derechos humanos suponen una política nacional e internacional racional y eficaz de desarrollo económico y social. 
Así, y en base a estos estándares, el CDESC concluye que el art. 11.1 del PIDESC representa una garantía que se debe entender de forma extensiva, en la medida en que constituye una pretensión de derecho fundamental que abarca un amplio conjunto de prestaciones materiales, sociales y territoriales, lo cual supone ir más allá, de la obligación que tiene Estado en orden a prevenir la falta de techo (FERRANDO NICOLAU E., 1992, p. 307). Esto significa, tal como comenta Espejo, que el derecho en estudio no se puede considerar de manera aislada, por el contrario, su interpretación y aplicación debe resultar congruente con el respeto, garantía y satisfacción de otros derechos humanos, y con el hecho que el acceso a una vivienda adecuada representa una condición necesaria para que las personas puedan desarrollar su plan de vida (ESPEJO YAKSIK, p. 53).

Ahora bien, es dentro de este contexto, donde se inserta el problema relativo a la integración territorial. En efecto, el CDESC parte de la base que la conexión que existe entre el espacio físico habitable y el entorno dentro del cual se ubica la vivienda, resulta determinante para asegurar el disfrute efectivo del derecho en comento (HOHMANN J., 2013, p. 179; GIL MC CAWLEY, 2015, p. 268). Por esta razón, y para cumplir con este objetivo, el Comité entiende que el Estado tiene, entre otras, la obligación de adoptar medidas que busquen reducir los problemas de segregación residencial que se puedan presentar dentro de su territorio. En concreto, el CDESC en su artículo 11 formula este mandato jurídico-objetivo en los siguientes términos:

La vivienda adecuada debe encontrarse en un lugar que permita el acceso a las opciones de empleo, los servicios de atención de la salud, centros de atención para niños, escuelas y otros servicios sociales. Esto es particularmente cierto en ciudades grandes y zonas rurales donde los costos temporales y financieros para llegar a los lugares de trabajo y volver de ellos puede imponer exigencias excesivas en los presupuestos de las familias pobres. De manera semejante, la vivienda no debe construirse en lugares contaminados ni en la proximidad inmediata de fuentes de contaminación que amenazan el derecho a la salud de los habitantes.

En general, con el término segregación, queremos aludir a la existencia de desiguales y a la separación de los sujetos en categorías que tienen una cierta base jerárquica o valorativa (RODRíGUEZ VIGNOLI, 2001, p. 13). Por tanto, en el caso del derecho a la vivienda, esto se traduce en que la segregación residencial viene a constituir un concepto con el cual se pretende hacer referencia a la distribución desigual de los grupos sociales en el espacio físico (WHITE M. J., p. 1009; KATZMAN R., 
2003, p. 19), o bien, "al grado en que dos o más grupos viven separados unos de otros, en diferentes partes del espacio urbano"(MASEEY \& DENTON, 1988, p. 282). En este sentido, debemos señalar que la segregación residencial está compuesta por tres dimensiones: en primer lugar, por la tendencia de un determinado grupo social a concentrarse en ciertas zonas; en segundo lugar, por la conformación de áreas socialmente homogéneas; y finalmente, por la percepción subjetiva que los habitantes de la ciudad tienen acerca de estos dos procesos (MASEEY \& DENTON, 1988, p. 282). Esta distinción tiene relevancia, puesto que llama la atención acerca del hecho que en realidad existen dos tipos de segregación, a saber: a) la segregación por localización de grupo, que es aquella forma de separación que tiene lugar cuando la gran mayoría de un grupo social vive en una determinada parte del territorio, y b) la segregación por exclusión, la cual atañe a la ausencia de mezcla o interacción entre distintos grupos sociales (RODRÍGUEZ VIGNOLI, 2001, p. 12). Sin embargo, no todo grupo que se encuentre distribuido de forma inequitativa en el espacio constituirá un grupo segregado. En efecto, nosotros razonamos en base a la idea que solo existirá segregación, cuando las opciones de localización o de acceso al suelo urbano que tengan estos colectivos se vean: "limitadas producto del accionar espontáneo u organizado, intencional o no- de otro u otros grupos. No hay segregación si estas restricciones no existen" (RODRÍGUEZ, G., 2014, p. 9). Esto significa que la segregación residencial nunca será estrictamente voluntaria, puesto que si esta no es en algún grado forzosa, entonces no será segregación, sino simplemente una forma de concentración territorial (RODRíGUEZ, G., 2014, p. 9.).

En lo que sigue, sostendremos que existen dos buenos argumentos para afirmar que el principio de integración territorial constituye un elemento que forma parte del contenido esencial del derecho a la vivienda, y que por tanto, los Estados tienen el deber ineludible de avanzar hacia la reducción de la segregación residencial. En seguida, precisaremos las obligaciones concretas que surgen para la administración en relación a esta dimensión del derecho en comento. Finalmente indicaremos porque es importante concebir a la integración territorial como un problema que debe ser abordado desde la perspectiva de los derechos humanos.

\section{2.- Razones a favor de considerar al principio de integración territorial como un elemento que forma parte del derecho a la vivienda}

En primer lugar, podemos distinguir en relación al derecho a la vivienda, dos dimensiones, una cuantitativa y otra cualitativa. La primera, se vincula con todos aquellos elementos que constituyen lo 
que podríamos llamar el núcleo material del derecho a la vivienda. En este contexto, una vivienda adecuada debe ser habitable, en el sentido de poder ofrecer espacio suficiente a sus ocupantes para protegerlos del frio, el calor, la lluvia, la humedad, el viento u otras amenazas para la salud (BRATT R., STONE M. \& HARTMAN C., 2006, p. 2), y contar de forma permanente con servicios de alcantarillado, agua potable, ventilación, alumbrado público o eliminación de residuos (CESCR, OBSERVACIÓN GENERAL N 4; 1991; ART. 11). La segunda dimensión, se relaciona con el lugar donde se ubica la vivienda. En este sentido, y tal como comentan Escobar y González, una vivienda no es adecuada, si esta se encuentra, por ejemplo, en una zona de difícil acceso o en el medio de un desierto, puesto que la misma también debe estar situada en un lugar que le permita a sus moradores poder tener acceso a opciones de empleo, salud, trabajo, educación o esparcimiento (ESCOBAR ROCA, 2012, tomo 2, p. 1302; HOHMANN J., 2013, p. 27). Esta afirmación tiene sentido, puesto que tal como explica Pisarello, el derecho a la vivienda constituye un derecho compuesto, en la medida en que su ejercicio resulta determinante para el disfrute de otras garantías fundamentales, tales como a la vida (ONU-FARHA L., 2016, PÁRR. 27), el derecho a la educación, al trabajo, a la salud física y mental, o al respeto a la vida privada y familiar (PISARELLO G., p. 259; HOHMANN J., 2013, p. 5.).

En este orden de ideas, y desde una perspectiva basada en el respeto a los derechos humanos, la falta de integración compromete las posibilidades que tienen las personas para poder salir de la pobreza, mejorar sus posibilidades de desarrollo y aumentar sus ingresos (SABATINI F., 2013, p. 16). Esto se explica en parte porque las familias que viven en zonas segregadas, tienen menores posibilidades para acceder a servicios públicos y privados de calidad, y también, a que presentan mayores dificultades para acumular algunas de las formas más útiles de capital social (ANDERSON E., 2010, p. 27). A este respecto, la segregación residencial constituye un fenómeno que impacta de manera negativa a los sectores menos aventajados, especialmente a través de sus resultados educacionales, lo cual se manifiesta en que los niños que viven en ciudades más segregadas, tienen menores posibilidad de acceder a la educación pre-escolar, presentan mayores índices de deserción escolar y mayores posibilidad de presentar retraso escolar que en comparación con los alumnos que viven en sectores más integrados (SANHUEZA C., p. 24; RODRIGUEZ J. \& ARRIAGADA C., 2004. p. 18). Del mismo modo, la falta de integración también compromete las posibilidades laborales de los miembros activos del núcleo familiar, en el sentido que estos suelen acceder a empleos de menor calidad, a que enfrentan mayor rotación laboral, y a que tienen mayores posibilidades de perder sus trabajos en relación con otros trabajadores que viven en conjuntos residenciales menos segregados 
(SABATINI F., 2013, p. 22; SABATINI F., CÁCERES G. \& CERDA J., p. 10; MOLINATTI F., 2013, p. 123). En esta misma línea, la segregación también dificulta que los sectores menos aventajados puedan crear lazos con personas de otras categorías sociales, lo cual resulta necesario para los efectos de establecer redes de contacto o colaboración que les permitan mejorar sus expectativas de desarrollo (KATZMAN R., 2003, p. 18). En definitiva, la falta de integración compromete la construcción de nuestra propia identidad, puesto que tal como apunta Hohmann, lo que es social, económica y culturalmente posible como forma de existencia, está en buena medida condicionado o determinado por el lugar donde vivimos (HOHMANN J., 2013, p. 178).

Por otro lado, en la medida en que la ciudad representa uno de los diversos espacios dentro de los cuales se despliega el poder y la soberanía del Estado, la provisión de vivienda también resulta crucial para la construcción de una sociedad democrática, entendida esta como una comunidad que está formada por sujetos que tienen intereses y problemas comunes (HOHMANN J., 2013, p. 218). Sin embargo, y tal como comenta Gil, la segregación constituye un obstáculo para el logro de este objetivo (GIL MC CAWLEY, 2015, p. 266). En efecto, la falta de integración favorece que los sectores más aventajados pierdan contacto y cercanía con los problemas que aquejan a las familias que viven en las zonas peor situadas, hecho que impide que aquellos puedan empatizar con la situación de estos últimos, lo cual genera un difícil de información y una pérdida de sentido comunitario (ANDERSON E., 2010, p. 98). En este sentido, el fenómeno en estudio también dificulta que las personas que viven en barrios segregados, puedan canalizar y articular sus demandas sociales de una forma más efectiva, por ejemplo, a través de procesos de participación ciudadana o por medio de la creación de grupos o comités habitacionales (ONU, 2018, PÁRR. 61.). Esta circunstancia compromete las posibilidades que tienen estos grupos para poder incidir en la marcha de los asuntos públicos en condiciones de igualdad, y para que sus requerimientos puedan ser atendidos por quienes detentan el poder político o económico (KATZMAN R., 2003, p. 18).

En otro orden de ideas, la segregación residencial también se vincula con el respeto al principio de igualdad y con la prohibición de toda forma de discriminación. En este sentido, debemos partir de la base que la segregación residencial puede ser tanto directa como indirecta (MARENGO M. \& ELORZA, A., 2018, p. 1547.). En el primer supuesto, esta tiene lugar cuando ciertas personas o grupos familiares son desplazadas de las mejores zonas de la ciudad aduciendo para ello alguno de los motivos prohibidos de discriminación, por ejemplo, en razón de su origen étnico o social (CESCR, OBSERVACIÓN 
GENERAL N²0; 2009; ART. 2). En el segundo caso, la segregación se verifica cuando los poderes públicos toman medidas que aparentemente son neutras o no discriminatorias, pero que en la práctica, le impiden de manera desproporcionada a ciertos grupos de la población el acceso a una vivienda bien ubicada (CESCR, OBSERVACIÓN GENERAL N²20; 2009; ART. 2). En este evento, la segregación indirecta se produce, por ejemplo, cuando se construyen complejos de viviendas en zonas periféricas de la ciudad, donde los costos que supone el uso del suelo son más bajos, o en lugares que no cuentan con servicios sociales o privados de buena calidad (LOPEZ MORALES, 2015, p. 199). En este contexto, la segregación residencial constituye un problema, puesto que deteriora la cohesión de grupo, favorece la reproducción se estigmas, y facilita la creación de guetos al interior de la ciudad (SABATINI, 2013, p. 16). En efecto, tal como indica Sabatini, las cifras muestran que el hecho de vivir en un conjunto segregado dificulta la búsqueda de empleo, eleva la vulnerabilidad social, destruye los lazos comunitarios, afecta los proyectos de vida de los más jóvenes y ayuda a guetizar los conjuntos de viviendas sociales (SABATINI F., 2013. p. 25 y ss.).

Por otro lado, este fenómeno afecta de manera especial a ciertos grupos de la población, considerados desde el punto de vista del derecho internacional, como especialmente vulnerables, tales como a los inmigrantes, a las personas con discapacidad, o a las mujeres:

> En el supuesto de los inmigrantes, estos se caracterizan por tener mayores dificultades para adquirir o arrendar viviendas de calidad, en barrios bien ubicados y provistos de servicios e instalaciones adecuadas (ONU-ROLNIK R., 2010, PÁRR. 30; ONU- FARHA L., 2016, PÁRR. 18), de forma que en muchos casos se ven obligados a vivir: "en zonas sin servicios ni planificación donde las condiciones son precarias y la infraestructura insuficiente, circunstancias que contribuyen a fragmentar las ciudades y fomentar la segmentación espacial”(ONU-ROLNIK R., 2010, PÁRR. 44).

> Las personas con discapacidad constituyen un colectivo particularmente vulnerable, dado que además de los problemas de salud que deben enfrentar debido a su condición, también deben soportar el hecho de ser víctimas de una serie de estereotipos y percepciones estigmatizadoras que ven a la discapacidad como una anormalidad o como una situación de inferioridad (ONUFARHA L., 2017, PÁRR. 12). Por esta razón, la ubicación de la vivienda resulta crucial, a efectos de poder tener acceso a servicios de salud, educación, trabajo o transporte adecuados, bienes que son determinantes para mejorar su calidad de vida (ONU- FARHA L., 2017, PÁRR. 56.). Sin embargo, 
los Estados no siempre toman en cuenta las necesidades de estas personas al momento de diseñar o implementar sus políticas de planificación urbana, lo cual provoca que muchas de ellas, las cuales al mismo tiempo pertenecen a los sectores de más bajos recursos, terminen viviendo en las zonas peor situadas de la ciudad (ONU-FARHA L., 2017, PÁRR. 56).

> En el caso de las mujeres, la segregación residencial tiene un impacto importante en materia de empleo, salud, educación, transporte y equipamiento comunitario (HOHMANN J., 2013, p. 185.). En este sentido, las jefas de hogar de las viviendas más segregadas presentan una mayor rotación laboral que los hombres en su misma situación, o que las jefas de hogar que pertenecen a los conjuntos habitacionales menos segregados, lo cual se explica en parte precisamente porque el costo y el tiempo de traslado que supone la existencia de ciudades altamente fragmentadas tiende a desalentar el empleo formal (SABATINI F., 2013, p. 22; ONU-ROLNIK R., 2011, PÁRR. 44). Por otro lado, la falta de integración territorial también se relaciona con los problemas de violencia domestica que afectan a millones de mujeres alrededor de mundo. A este respecto, y tal como sostiene Rolnik: "factores como el hacinamiento en las residencias, la mala habitabilidad y la falta de servicios accesibles (agua, electricidad y saneamiento) aumentan la incidencia de la violencia doméstica. Muchas mujeres en esta situación no pueden expulsar del hogar al autor, debido a la falta de apoyo de la familia, la comunidad y el Estado. Además, a muchas mujeres se les impide salir de las situaciones violentas porque no existe vivienda alternativa y apoyo financiero. Las que consiguen abandonar el hogar se vuelven vulnerables a la falta de hogar $y$, en consecuencia, pueden sufrir nueva violencia" (ONU-FARHA L., 2016, PÁRR. 24; ONU-ROLNIK R., 2011, PÁRR. 43).

Finalmente, y a luz de estas ideas, creemos que la segregación residencial constituye una demanda que debe ser abordada desde un enfoque basado en el respeto a los derechos humanos, toda vez que compromete el acceso a bienes públicos y privados en condiciones de igualdad, favorece la pérdida de cohesión social, pone en jaque la idea de democracia entendida como una comunidad de iguales, importa una forma de discriminación y marginalización tanto directa como indirecta y afecta de manera desproporcionada a los sectores más vulnerables de la sociedad.

\section{3.- Principales obligaciones que surgen para los Estados en relación al principio de integración $\underline{\text { territorial }}$}


El primer constitucionalismo se construye sobre la idea relativa a que los derechos representan facultades que se hacen valer en contra del Estado. De esta forma, el ente público asume un deber negativo: garantizar a todos una igual libertad jurídica y no intervenir en la esfera de autonomía de sus ciudadanos. Así, se asegura a los privados el derecho a negociar en igualdad de condiciones formales los términos de sus contratos, de manera que si alguno de ellos resulta perjudicado o ve desmejorada su posición material, dicha consecuencia solo es imputable a su propia incapacidad. Sin embargo, este modelo presenta algunos problemas. En efecto, la configuración de las relaciones de poder al interior de la sociedad, conduce a asimetrías entre aquellos que pueden efectivamente ejercer su libertad jurídica, y los que carecen de los medios para ello (BOCKENFORDE E., 1993). Para estos últimos, dicha libertad se transforma en una libertad inútil, dado que no cuentan con los presupuestos materiales para poder efectivamente ejercerla (GRIMM D., 2006, p. 163.). En este contexto, el Estado aparece ya no como el enemigo de la libertad, sino por el contrario, como su garante, de tal modo que asume la obligación consistente en desplegar un conjunto de actuaciones normativas e institucionales, destinadas a promover la esfera vital protegida por cada derecho fundamental (GRIMM D., 2006, p. 165.).

En el caso del derecho a la vivienda, estas ideas cobran una relevancia especial. En efecto, la garantía en comento satisface una necesidad básica (AÑON M. J., 1994.), de manera que su regulación no puede dejarse al simple al arbitrio del mercado o de la ley del contrato. Esto implica que el Estado tiene un margen de apreciación lo suficientemente amplio como parar impulsar medidas legislativas que vayan dirigidas a asegurar una mayor justicia social en la esfera de la vivienda, aun cuando dichas medidas interfieran con las relaciones contractuales entre particulares.

En relación con los desafíos relativos a la segregación residencial, nosotros creemos que el Estado tiene al menos dos obligaciones básicas, a saber, asegurar el buen uso del espacio urbano sobre una base de igualdad y no discriminación y facilitar, promover y garantizar la adecuada integración territorial de la ciudad.

\section{a) Asegurar el buen uso del espacio urbano sobre una base de igualdad y no discriminación:}

Como sabemos, la segregación puede tener su origen en políticas que de forma premeditada busquen expulsar a ciertos grupos de personas hacia los sectores peor situados del territorio urbano, 
en la existencia de planes de desarrollo territorial que no tomen en cuenta las necesidades de los sectores más vulnerables, en la falta de una adecuada red de transporte o de servicios sociales o comunitarios, en la ausencia de subsidios a la vivienda, o en prácticas discriminatorias que lleven a cabo empresas o individuos que pertenezcan al sector privado (ALARCÓN F., CISTERNA P., SILVA V. \& SCHONSTEINER J., 2016, p. 88). En la práctica, este fenómeno da lugar a lo que la doctrina llama, discriminación interseccional (ANDERSON, E., 2010, p. 64.). Con este concepto, se busca hacer referencia a la concurrencia de múltiples factores de discriminación, los cuales interactúan de tal forma que en conjunto, contribuyen a crear una situación de grave desigualdad o exclusión (EXPOSITO MOLINA, 2013. p. 210). En este sentido, y tal como afirma Rey Martínez: "no se trata sólo, un tanto asépticamente, de que se crucen dos factores (el género y la etnia, por ejemplo) en una situación concreta de discriminación simultánea, sino de valorar el hecho realmente crucial que de esa combinación de conjuntos o factores se genera un conjunto nuevo, distinto de los anteriores" (REY MARTINEZ, 2008, p. 266.). Ahora bien, como el principio de no discriminación constituye un estándar básico en materia de derechos humanos, creemos que resulta claro que el Estado tiene el deber de tomar medidas para avanzar hacia un reparto más equitativo del territorio (CESCR, OBSERVACIÓN GENERAL, N²0). Para lograr este objetivo, la administración debe establecer, al menos, un marco regulatorio que se haga cargo de las dificultades que conlleva la segregación residencial, tanto directa como indirecta, emplear incentivos o sanciones para alentar a los actores públicos y privados a modificar su actitud y su comportamiento frente a los individuos y grupos de individuos que son objeto de discriminación sistémica, y formular y ejecutar planes de acción, políticas y estrategias que abarquen de manera especial a los grupos más afectados por algunos de los motivos prohibidos de discriminación (CESCR, OBSERVACIÓN GENERAL N² 20; ONU-KOTHARI, 2008, PÁRR. 68-69-77.).

\section{b) Facilitar, promover y garantizar la adecuada integración territorial de la ciudad:}

Por otro lado, los Estados tienen la obligación general de cumplir con los derechos sociales, lo cual supone el deber de facilitar, promover y garantizar el disfrute efectivo de cada una de estas posiciones de derecho fundamental (ONU, 2018). Ahora bien, en el supuesto que nos ocupa, consideramos que esta obligación debe traducirse en la dictación de leyes que limiten la especulación inmobiliaria, que establezcan restricciones al uso del suelo, y que consagren la función social de la propiedad (ONU, 2018). Igualmente, creemos que este deber también se debe materializar en la entrega de subsidios que incentiven la construcción o el arrendamiento de viviendas ubicadas en zonas 
que cuenten con fuentes de empleo estable, escuelas de calidad, y buena conectividad. Del mismo modo, esta obligación debe comprender el deber de informar y dar publicidad a las leyes y disposiciones administrativas existentes en materia de vivienda, particularmente a aquellas que se refieren a la oferta disponible, a la situación de viviendas desocupadas y a la presencia de peligros medioambientales cercanos (ESPEJO YAKSIK, p. 58). Finalmente, el principio en estudio también debe abarcar la obligación de adoptar medidas para remediar los problemas de falta vivienda y segregación que caracterizan a los sectores o grupos más vulnerables de la población, lo cual conlleva el deber proporcionar la infraestructura física necesaria para que la vivienda sea considerada adecuada, y la obligación de que los distintos planes de desarrollo urbano incorporen una perspectiva basada en el respeto a los derechos humanos y al principio de interdependencia e indivisibilidad (ONU-HABITAT, 2010, p. 36.).

\section{4.- Importancia de concebir a la integración territorial, como parte del contenido del derecho a una vivienda adecuada}

En nuestra opinión, analizar la segregación residencial desde una perspectiva centrada en el respeto a los derechos humanos, conlleva cuatro consecuencias. En primer término, implica afirmar que los ciudadanos tienen el poder jurídico y social para exigir el cumplimiento de sus derechos relacionados con el disfrute de la garantía en comento (ABRAMOVICH V., 2006, p. 40.). Por esta razón, y a raíz de lo anterior, supone que la administración debe reconocer que las personas son titulares de una posición de derecho fundamental, y que por tanto, no deben ser tratadas como beneficiarias de servicios sociales que el Estado puede conceder o no según su voluntad. En este sentido, y tal como sostiene Farha:

Las personas que carecen de hogar o que habitan en viviendas inadecuadas se han considerado tradicionalmente como receptoras, beneficiarias u "objeto" de programas gubernamentales o caritativos. No obstante, cuando se reconoce su condición de titulares de derechos, pasan a ser sujetos activos, facultados para colaborar y participar en las decisiones que afectan a sus vidas y al goce de sus derechos. Esto significa que pueden contribuir a asegurar que las estrategias tengan en cuenta sus experiencias y, por lo tanto, sean más eficaces (ONUFARHA, L. 2018). 
En el mismo orden de ideas, también conlleva que la administración no puede actuar de manera discrecional o permanecer en la inactividad, toda vez que esta tiene el deber de ajustar su accionar a los principios y estándares que para estos efectos se establecen en el derecho internacional (ABRAMOVICH V., 2006, p. 40.). Finalmente, significa que la integración territorial debe constituir un principio que ha de informar todo el marco regulatorio relativo a la vivienda, en tanto representa una condición esencial para asegurar la igualdad, la dignidad y la autonomía de las personas, y también, porque permite que estas puedan desarrollar su plan de vida y perseguir sus propios objetivos de manera plena y sin discriminación.

\section{2.- La integración urbana en el derecho chileno: reconocimiento y análisis desde una perspectiva basada en el respeto a los derechos humanos}

\section{1.- Derecho urbano}

Lo que llamamos derecho urbano tiene una trascendencia invaluable. Claramente, no puede ser trivial la ordenación que afecta al lugar donde se estudia, trabaja, se divierte, se recrea, duerme, y esencialmente donde se vive, y se desarrollan los aspectos más esenciales de la vida. Explica lo anterior, el hecho de que cuando un precepto es "malo", rápidamente reciente la cotidianeidad de las personas, por ejemplo, bares autorizados en sectores residenciales, conjuntos habitacionales sin áreas de esparcimiento, puntos de congestión vehicular por falta de regulación, y la lista sigue.

Por otro lado, la mayoría de las personas comparten su sitio con otras, convivencia que da vida a las ciudades. Y es precisamente por esto, que el interés individual es imposible de considerar sin conciliarlo con el interés común o colectivo. Es la interacción entre dichos intereses la fuente desencadenante de conflictos. Justamente el derecho urbano buscará conciliar estos intereses a primera vista contrapuestos.

Pese a que la mayoría de la doctrina rechaza la autonomía del Derecho Urbano, ello no es obstáculo para reconocer su importancia, dado que salimos de una civilización rural para entrar en otra urbana, tal como señala García de Enterría, dejando la tierra de ser para el hombre un espacio natural de asentamiento, pasando a ser, un objeto más de su afán racionalizador. (CARCELLER A., 1989, p) 
De esta manera, la regulación urbanística recae básicamente sobre los inmuebles, sobre el suelo, y todos los usos posibles de aquel, generándose automáticamente un conflicto con el instituto de la propiedad, que también afecta a dicho bien.

Pero dicha reglamentación es compleja, por cuanto y como de buena forma lo ha señalado el Tribunal Supremo de España (2003, n. ${ }^{\circ}$ de recurso 4243/2000.):

La ciencia del urbanismo es esencialmente interdisciplinaria por confluir en ella conocimientos procedentes de las más variadas ramas del saber humano hasta el punto de que se considera ideal y deseable que dicha actividad sea realizada por un conjunto de profesionales -arquitectos, ingenieros, juristas, sociólogos, geógrafos, etc.- que sin orden de preferencia y bajo una dirección unitaria, colaboren en equipo aportando los conocimientos propios de sus respectivas actividades, y ello pone de manifiesto que la Ciencia Urbanística, en su estado actual, sobrepasa el ámbito específico de las titularidades tradicionales hasta el extremo de haber dado lugar a la nueva figura profesional del urbanista.

Y esta complejidad de lo urbano, no debe sorprendernos, ya que es el perfecto reflejo de la naturaleza de las personas, es una extensión de nuestra humanidad. Las ciudades, desde esta perspectiva, son entes vivos y en constante evolución, cambiando al ritmo en que lo hacen sus habitantes.

Por su parte ANTONIO CARCELLER FERNÁNDEZ (1989, p.23) define al derecho urbanístico como el "conjunto de normas jurídicas que, por sí mismas o a través del planeamiento que regulan, definen el contenido de la propiedad según su calificación urbanística y disciplinan la actividad administrativa encaminada a la urbanización y la edificación". El autor también le asigna a esta rama un contenido amplio ya que su regulación se lleva a cabo a través de un plan (propio de lo urbano), como sin él (incluyendo lo rural), con la importante salvedad de atribuirle a la legislación urbanística el poder de definir el contenido de la propiedad, cuestión discutible en nuestro país, ya que la Constitución habilita al derecho urbanístico por medio de la función social, para "limitar" la propiedad, no así para "definirla" -delimitar-, que es un concepto distinto; lo cual se explica por la diferente regulación constitucional existente entre ambos países en relación al derecho en cuestión.

La doctrina nacional representada por el profesor Ríos ha señalado que el derecho urbanístico es la técnica de aplicación de una actividad multidisciplinaria; esto es, la ordenación jurídica del 
planeamiento desarrollado sustantivamente por otras disciplinas: la arquitectura, la economía, la sociología, la geografía, etc. (RIOS L., 1985, p. 149.) De esta manera define al derecho urbanístico como "la juridización de normas de naturaleza técnica", provenientes del urbanismo. Sobre el particular se reproduce lo sostenido por el mismo autor en el sentido que "La necesidad del derecho urbanístico responde en definitiva a un llamado que el bien común, el orden público y la conciliación de los intereses privados hacen al legislador para impedir que la vida urbana sea más caótica de cuanto ha llegado a ser." (RIOS L., p. 161-164)

De forma y para cerrar el punto, podemos entender el Derecho Urbano como:

Un conjunto de normas y principios que regulan desde un punto de vista multidisciplinario, la ordenación racional del suelo, especialmente en lo relativo a la construcción; instituyendo los organismos competentes encargados de velar por su cumplimiento; y estableciendo las sanciones que se aplicaran en caso de contravención. (MORENO V., 2012, p. 43)

\section{2.- Autonomía.}

Un tema discutido en doctrina, es precisamente la autonomía relativa de esta rama, existiendo en derecho comparado distintas posiciones. Lo cierto es que es difícil sostener una independencia clara del área de estudio, pero también es innegable la notable relevancia teórica y práctica que ha ido adquiriendo y que pocos pensaron hace unas décadas, todo lo cual, hace presagiar que su atención, estudio y aplicación seguirá aumentando.

De esta forma hoy podemos constatar normas de naturaleza urbana claramente definidas, con objetivos propios y que necesariamente se basan valores determinados por la sociedad en la cual se imbuye. Es decir, existen principios, instituciones, materias y actores que perfilan, que dan forma a las normas urbanísticas.

Si bien en un primer suspiro, el derecho urbano estuvo enfocado en la regulación del derecho dominical, específicamente en las limitaciones al dominio en virtud de los requisitos que impone una adecuada convivencia social en la ciudad. Posterior a esto, y a medida que nuestra población se ha desarrollado, ya no solo económicamente, sino desde el punto de vista de otros valores, se ha visto como la legislación urbana, aun preocupada de la propiedad, ha ampliado su enfoque hacia nuevos 
intereses o demandas, como proteger el medio ambiente o desarrollo sustentable, lo cultural y arquitectónico de las construcciones, y por qué no agregar, el detentar una vivienda digna y de calidad.

\section{3.- Los Principios en general}

Es evidente y comprobable que el Derecho positivo no se encuentra en la posibilidad de prever y regular cada una de las posibles situaciones que puedan acontecer en la vida jurídica, especialmente en la rama específica que nos convoca, dotada de una especialización compleja en la que confluyen múltiples áreas del saber, como la arquitectura, la ingeniería, la economía, la política, el derecho etc.

Es por lo anterior que el mismo Derecho se encuentra habilitado para recurrir a fuentes diversas de la ley, a fin de dar solución a los problemas no previstos, como también, para extraer de ellos criterios interpretativos, limitativos, orientadores, entre otras funciones. Estas fuentes poseen un carácter abstracto, general y superior, que les permiten comprender aquellas situaciones particulares problemáticas.

Dichas fuentes no son otra cosa, que los denominados principios generales del derecho. Estos principios son para CARNELUTTI, la síntesis o condensación sistemática de las bases en que descansa un ordenamiento jurídico, de reglas no expresadas de las que derivan, a través de su aplicación a los casos previstos, los preceptos formulados con carácter general o particular. (VERA TAPIA, 2005, p. 18)

Los principios generales del Derecho se nos presentan como manifestaciones de los valores esenciales del hombre, y que éste percibe por el método de la inducción como también la deducción (PRIETO SANCHIS, 1992, p. 145), que poseen una existencia anterior al Derecho y que sobrepasan a los diversos ordenamientos jurídicos concretos, por tener un carácter atemporal y universal. (BOETSCH GILLET, 2011, p 18)

Estos valores cumplen distintas funciones, una por supuesto ya dicha, son las bases, el fundamento o inspiración normativa de una disciplina. Pero también cumplen funciones interpretativas e integradoras, cuyo análisis excede las finalidades del presente trabajo. 


\section{4.-Principios del derecho urbanístico}

El plan es el instrumento a través del cual se materializa la regulación urbana, normalmente sus principios se desarrollarán por medio de éstos. Pero, al ser instrumentos de corte administrativo deben dar ejecución a normas abstractas de jerarquía superior, en ese sentido los principios deben ser reconocidos por la legislación urbana en términos estrictos (llámese LGUC y OGUC), consagración que puede ser explícita o implícita, o bien, ser obtenidos por deducción o inducción.

En esta dirección, el artículo 2 de la Ley General de Urbanismo y Construcciones, establece que este cuerpo normativo "contiene los principios... que rigen a los organismos, funcionarios, profesionales y particulares, en las acciones de planificación urbana, urbanización y construcción."

Sin perjuicio de esta referencia, el mencionado cuerpo legal no enumera ni explicita a través de un título, párrafo o normas en particular aquellas directrices, por lo cual, habrá que deducir o inducir los principios del ordenamiento jurídico urbano.

En esta parte, siguiendo la doctrina positivista mayoritaria en nuestra tradición jurídica, sólo podremos determinar la existencia de un principio tan sólo después de analizar el ordenamiento positivo concreto, mediante el método de la deducción o de la inducción. En otras palabras, BOETSCH GILLET (2011, p. 19), citando a SQUELLA, nos dice que:

Las doctrinas positivistas sostienen que los principios generales del Derecho viven en cierto modo ínsitos en cada ordenamiento jurídico y pueden ser establecidos por medios inductivos a partir de las propias normas e instituciones del respectivo ordenamiento jurídico. Se trata, en suma, de componentes no escritos del ordenamiento jurídico, que viven en éste 'como el alcohol lo hace dentro del vino', y que informan sus normas e instituciones.

En su obra, LAUTARO RÍOS (1985, p. 262) considera como principios del Derecho Urbanístico: "legalidad, de la planificación legitimante, función social, igualdad, participación, auto tutela y de la protección jurídica de los administrados."

En esta línea y complementado al referido autor, podemos identificar algunos principios inspiradores del derecho urbano, tales como: el urbanismo como función pública, desarrollo urbano sustentable, participación ciudadana, legalidad, ordenación legitimante, equidistribución de cargas públicas, cooperación, e integración social. En lo que sigue, nos vamos a referir a este último principio. 


\section{5.- La integración social como principio en el derecho chileno}

La idea de integración, desde una perspectiva basada en el respeto a los derechos humanos, busca que los diferentes individuos que conforman nuestras ciudades o sociedad en general, formen parte activa del conjunto y gocen tanto de los beneficios como de las cargas que esta convivencia impone.

Desgraciadamente no es un valor predominante en nuestra convivencia, por lo cual su estudio impone necesariamente la obligación de hacer mención a su opuesto, que es la discriminación o segregación.

Esta última idea busca separar, excluir - con o sin intención- a personas de beneficios o de cargas sociales. Supone marginar e invisibilizar a aquellos grupos o personas que juzgamos como de segunda categoría o no aptos para una convivencia armónica, y que normalmente por razones en su mayoría económicas no puede oponerse a esta segregación, sino más bien, tácitamente se ven obligados a consentir en la misma.

La segregación es a la integración, lo que la discriminación es a la igualdad. Implica excluir, auto atribuirse el poder de considerar a otras personas o iguales, como de segundo orden, inferiores, y arbitrariamente no hacerlos participes de los beneficios de la vida en comunidad, perpetuando dichas diferencias en distintas esferas (residenciales, laborales, educativas, sanitarias, etc.). Determinando de buena forma, las oportunidades de desarrollo material y espiritual, de los ubicados en estratos bajos.

\section{5.- Causas de la segregación social residencial en el derecho chileno}

Entre las causas más relevantes que explican este fenómeno, encontramos las siguientes:

a) En primer término, y a pesar de la importancia que tienen los principios de igualdad y no discriminación, la integración no se ha visto favorecida ni impulsada por nuestra legislación urbana -al menos hasta ahora-. Es más, esta legislación ha sido usada de diferente forma e intensidad para segregar. En este sentido, podemos ilustrar muy bien y por desgracia esta situación a través del artículo 
55 de la propia Ley General de Urbanismo y Construcción (LGUC) al amparar la construcción de conjunto habitacionales sociales en sectores rurales; siendo consolidada dicha situación por medio de la Ley № 20.943, al permitir un equipamiento de mayor escala, fomentando el uso de esta normativa (PUCHEU M., 2004, REVISTA DERECHO UDEC n. ${ }^{\circ} 215-216$, p. 7-28).

b) Por este motivo, el resguardo de la integración social no ha sido fácil, a lo que debemos sumar la contribución que al caso han hecho el modelo de crecimiento por extensión o urban spraw (RAJEVIC E., 2010, p. 106-114) de las ciudades que se ha impuesto, basado en la utilización del automóvil como medio de transporte por excelencia, disminuyendo notablemente la posibilidad de encuentro entre diferentes sectores sociales, al estar dotadas cada agrupación de equipamientos y espacios que hacen innecesario la exploración de otras zonas de la ciudad. Estamos hablando de un modelo de urbanización de baja densidad, a diferencia del modelo Europeo, caracterizado por una urbanización concentrada, de alta densidad. Para estos efectos podemos citar el artículo 169 de la LGUC (introducido por la Ley № 20.958), que señala: Para los efectos de este Título se entenderá por crecimiento urbano por extensión el proceso que incorpora nuevo suelo urbanizado a consecuencia de un loteo; y por crecimiento urbano por densificación, el proceso que incrementa la intensidad de ocupación del suelo, sea como consecuencia del aumento de sus habitantes, ocupantes o edificación.

En definitiva, esta forma de desarrollo presenta un costo económico y social considerable, representado por el necesario transporte que debe existir entre las zonas residenciales que cada vez se alejan más de los centros urbanos, y que crean una peligrosa dependencia del automóvil, con impacto en la salud misma de una población cada vez más sedentaria, sin mencionar el costo ambiental que genera; y a su vez, crea una tendencia segregadora de fácil constatación, con zonas de alta plusvalía en contraste con otras muy alejadas y carentes de equipamiento y servicios elementales. En fin, como advierte de buena forma RAJEVIC, de todo lo anterior puede deducirse que no sólo estamos en presencia de una pérdida de contacto entre ricos y pobres; se trata de algo más profundo, de la erosión de la solidaridad social básica que necesita un Estado (RAJEVIC, 2010, p. 112).

c) En conexión con el punto anterior, al ser el suelo un recurso escaso, su demanda está determinada por su precio, respecto al cual la planificación local respectiva ha jugado un papel preponderante, ya que al asignar al suelo distintos usos y aplicar disímiles normas urbanísticas, lo afecta con diferente intensidad. Consagrándose sectores exclusivos de alta plusvalía versus otros rezagados como ya adelantábamos. 
Para este fin, un factor determinante ha sido la utilización del mercado como "institución eficiente" en la asignación de un recurso escaso como es el suelo urbano. Ya que si bien este coexiste excepcionalmente entre nosotros con el mecanismo del plan obligatorio (planes reguladores en general), en la práctica la regulación constitucional de la propiedad es muy vigorosa, lo que en definitiva, solo posibilita que el planificador vaya actuando conforme a los requerimientos del mercado, caracterizado por una débil participación ciudadana, no obstante las últimas reformas que tratan de enmendar aquello (Véase Ley 21.078).

Este es un grave inconveniente y difícil de superar, por cuanto, cualquier acción del Estado muy intensa sobre la propiedad urbana, puede ser considerada como una privación del derecho, la cual requiere obligatoriamente de una indemnización (expropiación), lo que hace inviable muchos proyectos de integración (como crear nuevos parques y espacios públicos en general o lograr mayor integración residencial), sin recurrir a la costosa expropiación para no incurrir en inconstitucionalidades (CPR, Art. $19 \mathrm{n} .{ }^{\circ} 24$ y 26). Justamente este uno de los fundamentos de la Ley № 20.958 que ya hemos nombrado y que busca establecer un sistema de mitigaciones y/ aportes que proyectos inmobiliarios públicos o privados obligatorios, para posibilitar un desarrollo más equilibrado entre lo inmobiliario, movilidad y espacios públicos, destacándose los aportes que deben efectuar obras por densificación, tradicionalmente exentas de las obligaciones de cesión derivadas del artículo 70 de la LGUC, no contribuyendo a generar espacio público a pesar de cambiar completamente la demografía de un sector.

d) A su vez, es llamativo ver que uno de los factores más decisivos en materia de segregación han sido las propias preferencias de las personas, manifestadas en lo que se ha llamado "usos de suelo localmente no deseados". Muchas personas son partidarias de mayores avances, enfocados en infraestructuras y equipamientos, pero, cuando estos comprometen su metro cuadrado su postura cambia. De esta forma han sido las propias personas las que fundadas en su interés propio han presionado a las autoridades locales a fin de que están establezcan regulaciones arbitrarias en zonas o sub zonas urbanas, regulando, por ejemplo, la altura de las edificaciones, la densidad máxima, tamaños mínimos de viviendas, excesivos requisitos para lotear, etc., con el fin implícito de reservar dichas áreas a determinados grupos sociales juzgados como homogéneos. 
A propósito de este tema, la literatura urbanística estadounidense ha acuñado los términos NIMBY y LULU. NIMBY es el acrónimo de la frase "not in my back yard", esto es, "no en mi patio trasero." LULU, el de "locally unacceptable land use", o sea, "usos de suelo localmente no deseados". En estos casos no es la necesidad del servicio lo discutido, sino su localización. Las razones esgrimidas son económicas, ambientales o de seguridad, ya que la mayoría de estos emplazamientos tendrán impacto en el valor de las viviendas ya apostadas, importarán externalidades negativas de contaminación; y no es un secreto que en general las zonas exclusivas no quieren en sus alrededores viviendas sociales, asociadas a grupos menos privilegiados o pobres, que se traducirán eventualmente en conflictos sociales o delincuencia, además de un menor valor de sus viviendas (SABATINI F., RASSE A., MORA P. y BRAIN I.; 2012, p. 179).

e) De igual forma, notable es la tendencia segregadora de permitir y demandar condominios tipo B, correspondientes a urbanizaciones privadas dispersas, muy similares a las fortificaciones medievales cuya finalidad era resistir invasiones externas (Ley n.o 19.537). En igual sentido, es palpable la tendencia a permitir cada vez con mayor frecuencia, los cierres de calles públicas por razones de seguridad.

Sobre esto último, se ha oscilado entre una postura primero restrictiva de la Contraloría General de la República, que rechazaba esta situación, por resultar incompatible con el uso común que respecto a ellos tienen todos los habitantes de la República, conforme los artículos 589 y 598 del Código Civil (Véase, Dictámenes №s 20.273/1993, 35.309/94, 28.870/1996, entre otros.), hasta una más amplia, que permite el enrejado, por cuanto el deber del Estado de promover el bien común, no sólo incluye asegurar a las personas el libre tránsito sino también su seguridad (Dictamen $\mathrm{N}^{\circ}$ 11.421/2000). Así, en la actualidad las municipalidades se encuentran facultadas por la LOCM -artículo 5 o letra c)- para "autorizar, por un plazo de cinco años, el cierre o medidas de control de acceso a calles y pasajes, o a conjuntos habitacionales urbanos o rurales con una misma vía de acceso y salida, con el objeto de garantizar la seguridad de los vecinos"( Véase en Chillán, Ordenanza que regula el cierre de calles pasajes y conjuntos habitacionales por motivos de seguridad ciudadana, Decreto Exento № 202/5719/2013, en relación con el articulo 5 letra c) de la Ley № 18.695, modificado por ley № 20.499 de 2011) 
El problema, es que estas autorizaciones posibilitan crear verdaderas "unidades" al modo de "condominios", pero carentes de regulación y portadoras de los mismos vicios, en cuanto a la idea de no integrar ni integrase a la comunidad, ni mitigar, ni contribuir, sino es más, reservándose espacio que de otra forma seria público.

f) Finalmente, merecen referencia - como factores de segregación- políticas públicas de antaño, inconfesadamente excluyentes, como las políticas de vivienda que privilegiaron la cantidad de hogares construidos por sobre su adecuada inserción en la trama urbana (admitiendo una suerte de "urbanismo de segunda categoría" para rebajar costes) o las políticas económicas que utilizan instrumentalmente la industria de la construcción, subordinando el urbanismo a sus propósitos. Por último, también deben observarse con cuidado otras políticas públicas como la educativa, que ha tenido discutibles efectos sobre la cohesión social, donde hoy son comunes colegios para distintas clases sociales.

\section{6.- Consagración de la integración social como principio en el derecho chileno}

En base a estas consideraciones, nos debemos preguntar si la integración social es realmente un principio que informa el derecho chileno, o bien, si solo constituye una directriz política, y por tanto, dependiente de los objetivos del Gobierno de turno. Al respecto, nuestra actual política nacional de desarrollo urbano la considera un principio, lo cual no garantiza su vigencia, salvo, que se constituya en un estándar que tenga un cierto reconocimiento o eficacia jurídica.

Lo anterior, podríamos sostener que ha ocurrido efectivamente en nuestros país, puesto que la planificación urbana debe orientar y regular el desarrollo de los centros poblados en función de la política nacional de desarrollo urbano. Por lo cual, los Instrumentos de Planificación Territorial respectivos, deben respetar dicho principio en su regulación.

Más allá de este débil reconocimiento, históricamente no ha existido una consagración legal con efectos prácticos, salvo que se sostenga que la integración social urbana es un principio derivado de otro mayor -lo cual tiene sentido-, cuál es el principio de igualdad. Por lo que sería un principio implícito en este último, lo que supone que este cumpliría una función inspiradora en la redacción de normas e interpretativa, pero no directamente integradora, sino solo a través del reconocimiento al 
principio de igualdad en nuestra Constitución.

Pero ¿por qué hablamos de uno u otro y no derechamente de igualdad? Creemos que podemos señalar que mientras la igualdad mira al individuo frente a los demás, la integración mira el interés de la sociedad en su conjunto. Así identificamos la segregación con la marginación de grupos que comparten ciertas características, y no solo de personas en particular. A lo cual se suma que la tradicional forma de entender el principio de igualdad como tratar de igual forma lo que es igual y de forma distinta lo que es distinto, no contribuye en la solución de nuestro problema de segregación, ya que justifica políticas y normas segregadoras, distintas según los sujetos.

También a través de la mentada la Ley № 20.958 podemos encontrar un reconocimiento del tema en estudio. Siendo particularmente relevantes los nuevos artículo 176, 183 y 184 de la LGUC, que reconocen explícitamente a la integración social como un objetivo primordial de la regulación urbana, textos que nos invitan a ver las consecuencias de su aplicación en un mediano plazo, frente a advertencias que hicieron en su oportunidad los urbanizadores privados, en cuanto a probables alzas en el precio de las viviendas a raíz de asumir costos de mitigaciones y/o aportes que antes no existían de la forma como los concibe esta ley.

Finalmente, siguiendo la tendencia de asumir la integración como un ideal a alcanzar, debemos consignar obligatoriamente a la Ley № 21.078 -actualmente en vigor-, la cual, entre muchos otros aspectos, agrega el nuevo artículo 28 decies a la LGUC, norma que establece que los planes reguladores deban ajustarse, entre otras cosas, a principios como la integración social (letra c). Lo cual deviene actualmente en un reconocimiento explícito a través de la propia ley sectorial del tema que nos convoca como un principio de la legislación urbana.

En sintonía con lo anterior, y en base a las ideas que hemos desarrollado en esta investigación, estimamos que la integración social en su variable residencial, impacta decididamente en la integración, interpretación, aplicación y fundamentación de las normas urbanísticas, que regulan el uso racional del suelo con diferentes intensidades, conforme al interés social, encontrando manifestaciones del principio de forma implícita en el reconocimiento del principio de igualdad contenido en diferentes normas constitucionales, de igual forma de diferentes normas contenidas en la LGUC podríamos desprender el valor en comento especialmente reconducidas a la política nacional 
de desarrollo urbano que contienen una consagración explicita de la integración como un principio y objetivo de la misma, en especial gracias a los aportes de las nuevas leyes números 20.958 de 2016, y 21.078 de 2018, con la salvedad que esta última efectúa un reconocimiento explícito esperemos de gran relevancia en lo sucesivo como principio.

En definitiva, creemos que en nuestra normativa, si existen suficientes elementos para concluir que la integración urbana constituye un principio que obliga a los poderes públicos a adoptar medidas con el objeto de avanzar hacia una distribución del espacio urbano sobre una base más equitativa. Sin embargo, este principio debe ser leído en función de los estándares de derecho internacional que hemos visto en la primera parte de este trabajo. Esto es así, puesto que la integración constituye una obligación que emana del contenido prescriptivo de un derecho humano, a saber, el derecho a vivienda, y no, de una simple política pública u objetivo social que el Estado puede o no perseguir. Solo de esta forma se pueda avanzar no solo hacia una mejor comprensión del derecho en comento, sino que, y esto es lo más relevante, hacia una más efectiva satisfacción de las necesidades habitacionales de los ciudadanos de nuestro país.

\section{7.- Medidas para favorecer la integración}

Por estas razones el contrato social ahora entendido como un contacto social ha ido desapareciendo. En la actualidad ni siquiera es un proceso que se manifieste al alcanzar una edad avanzada, sino que, comienza desde la cuna, es decir, niños "ricos" nacen, estudian y conforman sus redes, en lugares donde no existe la posibilidad de un contacto con los niños más "pobres", todo amparado por sus padres, quienes, por su propia formación, desconfían por diversas razones, del resultado que dicha convivencia puede generar.

Si bien nuestra generación puede notar esta separación, "tal vez" nuestros hijos no la perciban, porque su distanciamiento será - si no se evita- tan grande en relación a la otra parte o realidad de la sociedad, que su existencia y problemas les sean indiferentes e incluso contrapuestos, ya que todos sabrán que hay pobres, clase media o ricos, pero no habrán compartido entre ellos; no serán parte de sus dificultades o esperanzas, ni tampoco sentirán compromiso con el necesario auxilio que requieran; por lo cual malamente podrán unos entender las razones o móviles de las demandas de otros. Más aun, toda acción a favor de los más desfavorecidos, será fuertemente resistida y mirada como una 
simple carga por los otros.

La situación expuesta determina el fin del sentimiento de solidaridad de unos respecto de otros, cuestión elemental en una vida urbana cohesionada, de lo contrario, seguiríamos viviendo en la época feudal; sin perjuicio de que creo, cada vez nos asemejamos más, con instituciones como las mencionadas en puntos anteriores.

Creemos firmemente que, para superar el aislamiento social existente, se debe fomentar la creación de espacio público de calidad, donde el Estado y los privados tengan un rol protagónico, y en dicha "buena" dirección van las noveles leyes ya señaladas.

Es fácil constatar que el espacio público de calidad, con áreas verdes de calidad, equipamiento deportivo de calidad y en general zonas de esparcimiento de calidad, genera un beneficio social inconmensurable; versus el espacio público emanado del mercado, representado fielmente por los centros comerciales o malls, donde solo reproducimos el aislamiento visualizado, ya que la convivencia en estos espacios está determinada por orden de rentabilidad.

Otra medida, es replantearse el tipo de ciudad que queremos, si seguir creciendo en extensión o bien esforzarnos en pro de la integración y optar por modelos de densificación. O bien impulsar políticas de renovación de los centros urbanos, hoy abandonados en gran parte de nuestras ciudades. Lo cual claro tiene un costo importante, pero debemos consensuar en que su beneficio a largo plazo será mayor con una ciudad a escala humana y responsable con el medio ambiente.

Como corolario, estimamos que las formas para favorecer la integración no vienen dadas por imponer por parte del Estado una visión política expropiante. No es el medio idóneo quitar a los más beneficiados para entregar a los menos favorecidos, por cuanto ello fomenta resentimientos que solo perpetúan diferencias que se buscan evitar y cuyo fracaso global es del todo indiscutible.

La solución viene dada por favorecer el principio urbano de cooperación público-privado en la solución de un problema común. La segregación tarde o temprano nos afecta a todos.

Por ello, apuntan en una buena dirección las políticas públicas que no crean viviendas, centros 
de salud, establecimientos educacionales, etc., para los sectores más vulnerables; sino que insertan a los menos aventajados en el ambiente de los más favorecidos, siendo una carga contemplar en proyectos habitacionales -de calidad-, educacionales, entre otros, cupos o lugares destinados a familias emergentes. En dicha dirección apunta el proyecto de integración social en actual discusión legislativa, El cual en el papel busca permitir que más familias accedan a viviendas en barrios bien localizados y cercanos a servicios como transporte, salud, educación y áreas verdes. El proyecto contempla también incentivos normativos para la construcción de determinados esquemas a cambio de la incorporación de viviendas sociales, lo que permitirá que personas con subsidio estatal accedan a vivir en lugares donde antes no podían por el alto precio del suelo. Y por cierto de manera expresa es consagración de esta idea la ley № 20.958, ya comentada.

De esta manera, somos todos parte del mismo problema, y somos participes en la solución. Sin recurrir a ilegalidades, sino al rol subsidiario del Estado plenamente vigente en el marco de una economía de mercado.

\section{3.- Conclusión}

Tal como hemos visto en la primera sección de este trabajo, en el derecho internacional se establecen una serie de obligaciones de efecto progresivo, las cuales en general disponen que los Estados deben asegurar el buen uso del espacio urbano sobre una base de igualdad y no discriminación y avanzar hacia un mejoramiento de la calidad de vida de sus ciudadanos en términos del acceso equitativo al goce de aquellos servicios públicos o privados que resultan necesarios para que puedan llevar a cabo su plan de vida. Sin embargo, nuestro ordenamiento parte de la base que el mercado constituye el mecanismo más eficiente para asignar el uso del espacio urbano.

Este principio, el cual resulta válido y perfectamente legítimo en relación a la distribución de una gran cantidad de bienes y servicios, se ha revelado problemático en materia de derecho a la vivienda. En efecto, nuestro marco regulatorio, tal como comenta Gil Mc Cawley, restringe la asistencia estatal a un sector definido de la ciudanía, el cual no es capaz de participar en el mercado habitacional por sus propios medios, estimulando al sector privado, únicamente a través del sistema de subsidios a 
la vivienda, a entregar una solución habitacional a las familias de más bajos ingresos (GIL MCCAWLY, 2015, P.276). En la práctica, este mecanismo impide que los sectores más vulnerables de la población puedan acceder a una vivienda adecuada, con lo cual se favorece su exclusión y la separación territorial del espacio urbano.

Sin embargo, y a pesar de lo anterior, creemos que el mencionado artículo 28 decies, al reconocer a la integración social como un principio que debe informar el uso del suelo y la planificación urbana, abre la vía para que la administración pueda generar una política habitacional más acorde con la conceptualización del derecho a la vivienda como un derecho humano. A este respecto, nosotros proponemos que este principio debe ser interpretado a la luz de las obligaciones internacionales que ha asumido nuestro país en materia de derecho a la vivienda, puesto que esta la forma más adecuada de avanzar hacia la efectiva satisfacción de las necesidades habitacionales y de integración de nuestros ciudadanos.

\section{REFERENCIAS BIBLIOGRÁFICAS}

ABRAMOVICH, V. Una aproximación al enfoque de derechos en las estrategias y políticas de desarrollo. Revista de la CEPAL, no 88, 2006.

ALARCÓN, F; CISTERNA, P; SILVA, V. y SCHONSTEINER, J. Derecho a una vivienda adecuada en Chile: una mirada desde los campamentos. Informe Anual sobre Derechos Humanos en Chile 2016. Centro de Derechos Humanos UDP. Santiago, Chile: Ediciones Universidad Diego Portales, 2016.

ANDERSON, E. The imperative of integration. Princeton, Nueva Jersey, Estados Unidos: Princeton University Press. 2010.

AÑON, M.J. Necesidades y Derechos. Un ensayo de fundamentación. Madrid, España: Centro de Estudios Constitucionales. 1994. 
BOCKENFORDE, E. Escritos sobre derechos fundamentales. Baden-Baden: Nomos, 1993, 1986.

BOETSCH GILLET, Cristian (2011): La buena fe contractual. Editorial jurídica de Chile, Santiago de Chile, p.18 y 19.

BRATT, R.; STONE, M. y HARTMAN, C. A Right to Housing: Foundation for a New Social Agenda. Philadelphia: Temple University Press (p. 2). 2006.

COMITÉ DE DERECHOS ECONÓMICOS SOCIALES Y CULTURALES. Observación General N 4: El derecho a una vivienda adecuada. Art. 11, 1991.

COMITÉ DE DERECHOS ECONÓMICOS, SOCIALES Y CULTURALES. Observación General N²0: La no discriminación y los derechos económicos, sociales y culturales (artículo 2, párrafo 2 del Pacto Internacional de Derechos Económicos, Sociales y Culturales). 2009.

CARCELLER, A. (1989): Instituciones de derecho urbanístico. Editorial Montecorvo S.A., Madrid, España, p. 23 y 27, citando a García De Enterría.

Constitución política de la república de Chile, articulo 19 números 24 y 26.

Declaración De Teherán: Art. 13. 1968.

ESCOBAR ROCA. G (ed.). Derechos sociales y tutela antidiscriminatoria. Tomo 2, p. 1302. Aranzadi, Pamplona. 2012.

ESPEJO YAKSIK, N. El derecho a una vivienda adecuada Centro de Investigación Social: Un Techo para Chile, año 8, Número 13. 58.

EXPOSITO MOLINA, C. ¿Qué es eso de la interseccionalidad? Aproximación al tratamiento de la diversidad desde la perspectiva de género en España. Investigaciones Feministas. 2013.

FERNÁNDEZ RUIZ-GÁLVEZ, M. E. Anuario de Filosofía del Derecho, N XIV. Derechos humanos: ¿yuxtaposición o integración? (p. 696). 1996. 
FERRANDO NICOLAU, E. Anuario de filosofía del derecho, № 9. El derecho a una vivienda digna y adecuada.1992.

GIL Mc Cawley, D. La desigualdad: Seminario en Latinoamérica de Teoría Constitucional y Política. Derecho a la vivienda, integración urbana y minimalismo regulatorio en Chile. Buenos Aires, Argentina: Ediciones Libraria, 2015.

GRIMM, D. Constitucionalismo y derechos fundamentales. Madrid, España: Trotta, 2006.

GIL MC CAWLEY (2015): derecho a la vivienda minimalismo regulatorio en Chile, p. 276.

HOHMANN, J. The right to housing: Law, Concepts, Possibilities. Oxford: Hart Publishing. 2013.

KATZMAN, R. La dimensión espacial en las políticas de superación de la pobreza urbana. CEPAL. Santiago: Serie: Medioambiente y desarrollo, n59, 2003.

LOPEZ MORALES, E. Suelo urbano y segregación residencial: hacia una agenda de integración social para zonas centrales metropolitanas chilenas. Ciudades, n 18, p. 199. 2015.

Ley №21.078 (15 de febrero de 2018) sobre transparencia del mercado del suelo e impuesto al Aumento de valor por ampliación del límite urbano.

Ley № 19.537 sobre Copropiedad Inmobiliaria.

MASEEY, D. y DENTON, N. The Dimensions of Residential Segregation. Social Forces. Vol. 67, $n^{\circ} 2$. 1988.

MOLINATTI, F. Segregación residencial socioeconómica en la ciudad de Córdoba (Argentina): tendencias y patrones espaciales. Revista INVI, Vol. 28, nº 79. 2013.

MARENGO, M., ELORZA, A. Segregación residencial socioeconómica y programas, habitacionales públicos: el caso del programa mi casa mi vida en la ciudad de Córdoba, Argentina. Revista de Direito da Cidade, $n^{\circ} 10, N^{\circ} 3,2018$. 
MORENO, V. (2012): El ius aedificandi. Su relación con la institución dominical y urbanistica. Memoria para optar al grado de licenciado en ciencias juridicas y soliciales, Universidad de Concepción. p. 54.

NACIONES UNIDAS - KOTHARI, M. Informe del Relator Especial sobre la vivienda adecuada como elemento integrante del derecho a un nivel de vida adecuado y sobre el derecho de no discriminación a este respecto: 2008 .

NACIONES UNIDAS- FARHA, L. Informe de la relatora especial sobre el derecho a la vivienda adecuada como elemento integrante del derecho a un nivel de vida adecuado: 2016.

NACIONES UNIDAS- FARHA, L. Informe de la Relatora Especial sobre una vivienda adecuada como elemento integrante del derecho a un nivel de vida adecuado y del derecho de no discriminación en este contexto. 2017 y 2018.

NACIONES UNIDAS. Informe de la Relatora Especial sobre una vivienda adecuada como elemento integrante del derecho a un nivel de vida adecuado y sobre el derecho de no discriminación a este respecto. 2018.

NACIONES UNIDAS-ROLNIK, R. Informe de la Relatora Especial sobre una vivienda adecuada como elemento integrante del derecho a un nivel de vida adecuado y sobre el derecho de no discriminación a este respecto. 2008, 2010 y 2011.

NOGUIERA, H. Diálogo judicial multinivel y principios interpretativos favor persona y de proporcionalidad. C, NASH. El principio pro persona en la jurisprudencia de la Corte Interamericana de Derechos Humanos (p. 170). Santiago, Chile: Librotecnia. 2013. CONVENCIÓN DE VIENA SOBRE EL DERECHO DE LOS TRATADOS: Art. 31. 1969. (entrada en vigor: 27 de enero de 1980).

ONU-HABITAT. El derecho a una vivienda adecuada. 2010.

PACTO INTERNACIONAL DE DERECHOS ECONÓMICOS, SOCIALES Y CULTURALES: Art. 11.1. 1966 (entrada en vigor: 3 de enero de 1976). 
PRIETO SANCHÍS, Luis (1992): Sobre principios y normas. Problemas del razonamiento jurídico. Editorial Centro de Estudios Constitucionales, España, p. 145.

PUCHEU, M. (2004): “El Uso del Suelo Rustico. ¿Prohibición de Construir o Prohibición de Cambiar el Destino Agrícola?". Revista de Derecho, Universidad de Concepción (Número 215-216, págs. 7-28) Concepción, 2004.

PISARELLO, G. El derecho a la vivienda: constitucionalización débil y resistencias garantistas. Chapecó, v. 14, $\mathrm{n}^{\circ} 3.2013$.

PISARELLO, G. Vivienda para todos: derecho en construcción. p. 259. Recuperado de https://archivos.juridicas.unam.mx/www/bjv/libros/10/4556/12.pdf.

PINTO, M. La aplicación de los tratados sobre derechos humanos por los tribunales locales. El principio pro homine: Criterios de hermenéutica y pautas para la regulación de los derechos humanos (p. 163). Buenos Aires: Editores del Puerto. 1997. Pacto Internacional De Derechos Civiles Y Políticos: Art. 5.2. 1966 (entrada en vigor: 23 de marzo de 1976).

RAJEVIC, E. Cohesión social e intervención administrativa el territorio urbano (Con Especial Referencia A España Y Chile) Departamento de Derecho Público del Estado, Universidad Carlos III de Madrid, p. $106-114$ y 281.

RODRÍGUEZ VIGNOLI, J. Segregación residencial socioeconómica: ¿Qué es? ¿Cómo se mide? ¿Qué está pasando? ¿lImporta? CEPAL. Santiago: Serie: Población y desarrollo, nº 16, 2001.

RODRÍGUEZ, G. Biblio 3W, Revista Bibliográfica de Geografía y Ciencias Sociales, XIX. Qué es y qué no es segregación residencial. Contribuciones para un debate pendiente. 2014.

REY MARTINEZ, F. La discriminación múltiple, una realidad antigua, un concepto nuevo. Revista española de derecho constitucional, $n^{\circ} 28$ (84). 2008. 
RIOS, L. (1985), Ob. Cit. Nota № 1, p. 149, 161-164 y 262.

RAJEVIC, E. (2010): "Cohesión social e intervención administrativa en el territorio urbano (con especial referencia a España y Chile). Tesis Doctoral, dirigida por el sr. Luciano Parejo

SABATINI, F. Documento del Instituto de Estudios Urbanos, $n^{\circ} 29$. Tendencias de la segregación residencial urbana en Latinoamérica: Reflexiones a partir del caso de Santiago de Chile. 1999.

SABATINI, F. Segregación de la vivienda social: ocho conjuntos en Santiago, Concepción y Talca. Segregación de la vivienda social: reducción de oportunidades, pérdida de cohesión. Santiago, Chile: Colección Estudios Urbanos UC, 2013.

SABATINI, F.; CÁCERES, G.y CERDA, J. Segregación residencial en las principales ciudades chilenas: tendencias de las tres últimas décadas y posibles cursos de acción. Revista EURE, vol., 27, nº 82.

SANHUEZA, C. Residential Segregation Effects on Poor's Opportunities in Chile. p. 24. Recuperado de http://repositorio.uchile.cl/handle/2250/144350. RODRIGUEZ, J. y ARRIAGADA, C. Segregación residencial en la ciudad latinoamericana. Revista EURE, vol. XXIX, nº 89. 2004.

Sentencia del Tribunal Supremo de España, sala 3ạ, de lo contencioso-administrativo, 8 de mayo de 2003 , numero de recurso $4243 / 2000$.

SABATINI, Francisco; RASSE, Alejandra; MORA, Pía y BRAIN, Isabel (2012): Ob. Cit. Nota № 128, p. 179.

VERA TAPIA, Carla Andrea (2005): “Gestión y organización administrativa en el derecho urbanístico". Memoria de prueba para optar al grado de licenciado en ciencias jurídicas y sociales, Universidad de Concepción, Concepción - Chile, p. 18.

WHITE M. J. American Journal of. Sociology, $n^{\circ} 88$. The measurement of spatial segregation (p. 1009). 
Trabalho recebido em 04 de novembro de 2019

Aceito em 30 de janeiro de 2020 